\title{
Effects of low-carbohydrate diet therapy in overweight subjects with autoimmune thyroiditis: possible synergism with ChREBP
}

This article was published in the following Dove Press journal:

Drug Design, Development and Therapy

14 September 2016

Number of times this article has been viewed

\author{
Teresa Esposito ${ }^{1,2}$ \\ Jean Marc Lobaccaro ${ }^{3}$ \\ Maria Grazia Esposito ${ }^{4}$ \\ Vincenzo Monda' \\ Antonietta Messina' \\ Giuseppe Paolisso ${ }^{5}$ \\ Bruno Varriale ${ }^{2}$ \\ Marcellino Monda' \\ Giovanni Messina ${ }^{1,6}$ \\ 'Department of Experimental \\ Medicine, Section of Human \\ Physiology and Unit of Dietetics \\ and Sports Medicine, ${ }^{2}$ Laboratory \\ of Molecular Biology and Genetics, \\ Department of Experimental \\ Medicine, Second University of \\ Naples, Naples, Italy; ${ }^{\mathrm{U}} \mathrm{UMR}$, Clermont \\ Université, Centre de Recherche \\ en Nutrition Humaine d'Auvergne, \\ Aubière Cedex, France; ${ }^{4}$ Complex \\ Surgery Unit, Evangelic Hospital Villa \\ Betania, ${ }^{5}$ Department of Scienze \\ Mediche, Chirurgiche, Neurologiche, \\ Metaboliche e dell'Invecchiamento, \\ Second University of Naples, \\ Naples, ${ }^{6}$ Department of Clinical and \\ Experimental Medicine, University of \\ Foggia, Foggia, Italy
}

Correspondence: Giovanni Messina Department of Clinical and Experimental Medicine, University of Foggia, Via L Pinto, 7 I I 22 Foggia, Italy

Tel +39 88I 5665804

Fax +39 88I 5665844

Email gianni.messina@unina2.it

\begin{abstract}
The thyroid is one of the metabolism regulating glands. Its function is to determine the amount of calories that the body has to burn to maintain normal weight. Thyroiditides are inflammatory processes that mainly result in autoimmune diseases. We have conducted the present study in order to have a clear picture of both autoimmune status and the control of body weight. We have evaluated the amount of either thyroid hormones, or antithyroid, or anti-microsomal, or anti-peroxidase antibodies (Abs) in patients with high amounts of Abs. In a diet devoid of carbohydrates (bread, pasta, fruit, and rice), free from goitrogenic food, and based on body mass index, the distribution of body mass and intracellular and extracellular water conducted for 3 weeks gives the following results: patients treated as above showed a significant reduction of antithyroid ( $-40 \%, P<0.013)$, anti-microsomal $(-57 \%, P<0.003)$, and anti-peroxidase $(-44 \%$, $P<0,029)$ Abs. Untreated patients had a significant increase in antithyroid $(+9 \%, P<0.017)$ and anti-microsomal $(+30 \%, P<0.028)$ Abs. Even the level of anti-peroxidase Abs increased without reaching statistical significance $(+16 \%, P>0064)$. With regard to the body parameters measured in patients who followed this diet, reduction in body weight $(-5 \%, P<0.000)$ and body mass index $(-4 \%, P<0.000)$ were observed. Since $83 \%$ of patients with high levels of autoantibodies are breath test positive to lactase with a lactase deficit higher than $50 \%$, this fact led us to hypothesize a correlation with carbohydrate-responsive element-binding protein and therefore a possible role of carbohydrate metabolism in the development and maintenance of autoimmune thyroiditis associated with body weight increase and slower basic metabolism.
\end{abstract}

Keywords: thyroiditis, thyroid hormones, thyroid autoantibodies, low-carbohydrate diet, protein diet

\section{Introduction}

The term thyroiditis refers to a group of disorders characterized by inflammation of the thyroid gland. There are several types of thyroiditis, and these can be generally classified according to their etiology or clinical course. So, from a practical point of view, thyroiditis can be subdivided into acute, subacute, and chronic thyroiditis. If this classification has the advantage of simplicity, it is obviously incomplete, because within each of these categories it is possible to recognize more specific clinical conditions, such as thyroiditis of the elderly. Today, Hashimoto's disease, also known as chronic lymphocytic thyroiditis, is one of the most diffuse clinical conditions affecting the thyroid gland throughout the world, especially in those geographic areas where iodine intake is particularly elevated. ${ }^{1}$ It is more common in women, and its incidence increases with age. Hashimoto's thyroiditis, an acute immune condition (aggression of the thyroid gland by the body's own immune system), generally tends to evolve 
toward hypothyroidism; it can, however, present with different signs or symptoms depending on the clinical course, age of onset, and other concomitant pathologies. In the vast majority of cases, Hashimoto's thyroiditis has an insidious onset and thyroid hormone levels usually remain within the normal range, with the first symptoms appearing only after the disease has progressed toward overt hypothyroidism. Only occasionally patients present with acute onset, and in these cases the symptoms are typically those of hyperthyroidism (irritability, fatigue, weight loss, rise of body temperature, etc.).

In patients with Hashimoto's thyroiditis, antithyroperoxidase antibodies (TPO Abs; >95\%) are usually detectable, while a minor percentage of patients $(60 \%)$ have also anti-thyroglobulin Abs. Anti-microsomal Abs also have an important role in Hashimoto's thyroiditis; even though the test is not sufficiently specific to be considered diagnostic, it does indicate the involvement of the immune system and inflammation. ${ }^{2,3}$ In doubtful cases, a definite diagnosis can be established with sonography and fine needle aspiration cytology. In western countries, almost half of the women population discover during their lifetime to have a thyroid disorder and all of the associated clinical symptoms. None the less, the official medical approach to the problem is overly simplistic, being it limited to monitoring the situation with periodic lab tests, while nothing else is done during the initial stages of the disease and the treatment, based exclusively on the administration of 1-thyroxine sodium, begins only upon the appearance of the first signs of thyroid insufficiency.

An important fact to be aware of is that there are goitrogenic foods that contain substances that interfere with iodine metabolism. ${ }^{4}$ These substances (known as goitrogens) are found especially in products of the cruciferous (Brassicaceae) family (rape seed or canola, cabbage, turnip, watercress, arugula, radish, horseradish) and in milk produced by cattle nourished with these vegetables. Other goitrogens include soy, spinach, millet, tapioca, and lettuce. Even certain food additives, ie, nitrates used for fish and meat preservation, prevent iodine uptake by the thyroid gland. These facts induce iodine deficiency which can cause hypothyroidism and the formation of goiter (abnormal enlargement of the thyroid). This is why these substances are called goitrogens. From a chemical point of view, the substances contained in these goitrogens are glucosinolates. Actually, the suppression of thyroid gland function, because of the interference with iodine metabolism, is due to the breakdown products of these glucosinolates, such as thiocyanate, isothiocyanate, and goitrin. Generally, these compounds are inactivated by cooking and their iodine metabolism antagonizing activity can be considered insignificant. By contrast, the presence of autoantibodies (anti-thyroglobulin Abs, anti-peroxidase Abs, and anti-microsomal Abs) can be considered "sentinel" signs in the vast majority of patients with Hashimoto's thyroiditis. Also, Basedow-Graves disease and a transitional form of thyroiditis following a postpartum or subacute thyroiditis (de Quervain's thyroiditis) show these "sentinel" signs. Iodine is essential for the production of thyroid hormones triiodothyronine and thyroxine. Triiodothyronine is by far the more biologically active thyroid hormone. It has a central role in the regulation of cell metabolism, including lipid and carbohydrate metabolism. ${ }^{5}$ An excessive intake of carbohydrates leads to deposits of adipose tissue and insulin resistance. In turn, glucose and insulin together regulate the de novo lipogenesis and the activation of various transcription factors, including SRBP1 and LXR. It has also been recently identified a carbohydrate response element (Chore) within the promoter of the hepatic L-type pyruvate kinase ( $L-P K)$ gene in rats. The target genes of the carbohydrate-responsive element-binding protein (ChREBP) are implicated in glycolysis, lipogenesis, and neoglucogenesis. ${ }^{6,7}$

In the current study, we have focused our attention on patients with autoimmune thyroid disorders, chronic Hashimoto's thyroiditis. In these subjects, a low-calorie diet, although well balanced in lipid, carbohydrate, and protein content, is not always successful, especially in terms of body weight reduction and decrease of autoantibodies titers. We have undertaken this study to investigate the whole endocrine status and the levels of autoantibodies in these patients in order to be able to create a personalized diet plan aimed at correcting not only body weight but also the levels and the effects of autoantibodies.

\section{Materials and methods}

In the current study, we enrolled a total of 180 patients, 84 males and 96 females, aged 30-45 years. All the subjects did not present celiac disease, but Hashimoto's disease was detected. Moreover, each patient showed others autoimmune symptoms, they are typical Hashimoto's disease. All the patients were monitored weekly, since it has been shown that this procedure improves the patient's physician empathy and consequently the achievement of a good result in those that followed the $\operatorname{diet}^{8}{ }^{8}$

Women in menopause were excluded. All the patients had increased levels of antithyroid Abs at baseline. The participants were provided with both written and oral information regarding the study protocol and were ensured that they were free to withdraw from the study at any time. All the subjects gave written informed consent before participation. All procedures conformed to the directives of the 
Declaration of Helsinki. This study was approved by the Azienda Universitaria Policlinico of the Second University of Naples, prior consultation with the ethics committee of the same Azienda Universitaria Policlinico, which issued the ethics code RDn 1185 of July 27, 2011, subsequently integrated with Decree 922 October 22, 2013.

Serum levels of free triiodothyronine (FT3), free thyroxine (FT4), and thyroid-stimulating hormone (TSH) were measured using the Benvenga and Trimarchi method. ${ }^{9}$ All the materials necessary were furnished by DiaSorin (Saluggia, Italy). In all the patients, levels of anti-microsomal, antithyroglobulin, and anti-peroxidase Abs were measured as suggested by the manufacturer (DiaSorin).

Body composition was determined by conventional body impedance analysis (BIA) with a single-frequency $(50 \mathrm{kHz})$ bioelectrical impedance analyzer (BIA $101 \mathrm{RJL}$; Akern Bioresearch, Firenze, Italy), according to the standard tetrapolar technique, with the subject in supine position and the electrodes placed on the dorsal surface of the right foot and ankle and right wrist and hand. The patients were asked to refrain from strenuous exercise and to maintain their usual intake of caffeinated beverages during the 3 days preceding the measurements. After overnight fasting, the patients were asked to empty their bladder before being evaluated. Body composition was then calculated by bioelectrical measurements and anthropometric data using the software provided by the manufacturer, which incorporated validated predictive equations for total body water, fat mass, and fat-free mass. All women were submitted to the BIA between the eighth day and 11th day from the beginning of menstrual cycle and they had not taken contraceptives in the last 3 months. All the participants had been fasting for 12 hours, and they had not consumed drinks for 4 hours; this condition assured an optimal state of hydration for BIA.

A total of 108 patients (44 males and 64 females) (experimental group) started a diet program based on the following proportions: carbohydrates $12 \%-15 \%$, proteins $50 \%-60 \%$, and lipids $25 \%-30 \%$. These patients were instructed to eat large leafy and other types of vegetables and only lean parts of red and white meat, avoiding goitrogenic food. The following items were also excluded from the diet: eggs, legumes, dairy products, bread, pasta, fruits, and rice. This protein-rich diet plan was implemented for 3 weeks, at the end of which bioimpedance tests, bodyweight measurements, and blood tests (TPO Ab, anti-microsomal Ab, thyroglobulin [TG] Ab, and thyroid hormones) were performed. The remaining 72 patients (40 males and 32 females) (control group) followed a simple, low-calorie diet without restrictions regarding the types of food to consume, but adhered to the recommended dietetic allowances, as suggested by the National Research Institute on Food and Nutrition. After 3 weeks, the same tests were performed in this group of patients as those performed in the treatment group.

\section{Statistical analysis}

Comparison of the data obtained was done using Student's $t$-test. The two groups in the study, the one receiving a protein-rich diet and the other a standard diet, were also compared using a two-tailed Student's $t$-test for independent data and heterogeneous variance. In the patients whose diet was devoid of goitrogenic foods, the correlation between hormone concentrations, Ab titers, and other physical parameters was also calculated using Spearman's rank-order correlation test; Spearman rank-order correlation coefficient is a nonparametric measure of correlation, because the values being compared are not homogeneous for type and variance. For all tests, significance was set at $P<0.05$ and was calculated using SPSS software.

\section{Results}

Initial (pre-diet) levels of FT3, FT4, and TSH were not significantly different between the two groups. Furthermore, in both the groups a nonsignificant variation of FT3, FT4, and TSH was observed after 21 days of diet regimen (Figure 1). As far as physical parameters are concerned, in the no-restriction, low-calorie diet group, only body mass index (BMI), lean mass weight, and body weight showed statistically significant variations from baseline $(-4 \%$, $P<0.000 ;-4 \%, P<0.000$; and $-5 \%, P<0.000$, respectively) after 21 days of diet regimen (Figure 2). Moreover, these two parameters (lean mass weight, and body weight) showed only a slight correlation with circulating levels of autoantibodies. More particularly, we observed an inverse correlation between pre-diet antithyroid $\mathrm{Ab}$ levels and body weight reduction ( $\mathrm{Rs}-0.659, P<0.015)$, a direct correlation between pre-diet levels of anti-microsomal Abs and BMI reduction (Rs $-0.687, P<0.045$ and Rs $-0.795, P<0.011$, respectively), and an inverse correlation between post-diet antithyroid Abs and a body weight reduction (Rs -0.608 , $P<0.028$ ) (not shown).

Patients having anti-TG, anti-microsomal, and antiTPO Ab but who did not follow a restricted dietary regime (exclusion of the goitrogenic food mentioned above) showed a significant increase in autoantibody levels, in particular anti-TG $\mathrm{Ab}(+9 \%, P<0.017)$ and anti-microsomal $\mathrm{Ab}$ $(+30 \%, P<0.028)$. Anti-TPO Abs showed a slight but not significant increase $(+16 \%, P<0.004)$ (Figure 3$)$. By contrast, patients showing high concentrations of anti-thyroglobulin 

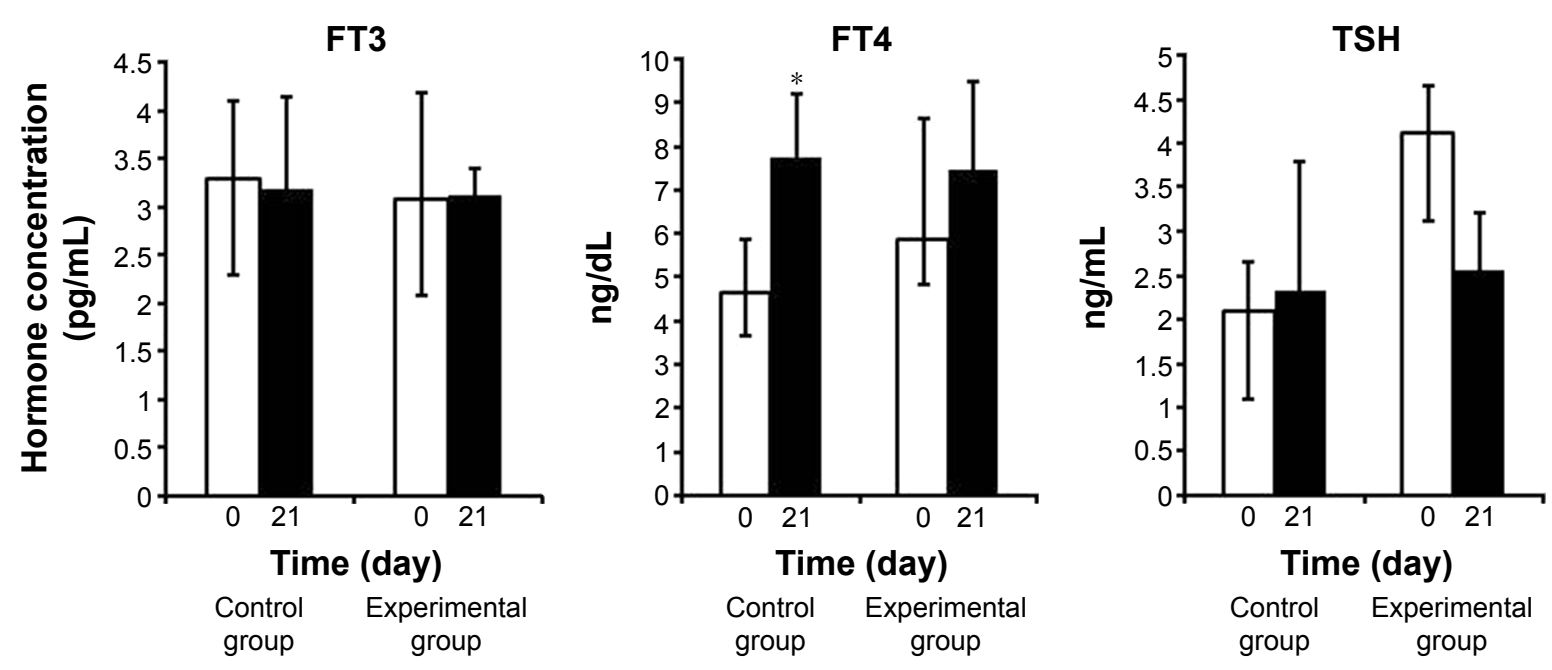

Figure I Hormonal status of FT3, FT4, and TSH in patients of both the groups (control and experimental).

Notes: Only in the control group, a slight significant increase in FT3 was noticed after 21 days of the diet regimen. Open bars indicate the patients at the beginning of experimental time, while the solid bars indicate values of patients after 21 days of the diet. *Significance of differences was set at $P<0.05$.

Abbreviations: FT3, free triiodothyronine; FT4, free thyroxine; TSH, thyroid-stimulating hormone.

Abs, anti-peroxidase Abs, and anti-microsomal Abs were suggested to follow a specific diet that was devoid of carbohydrates, dairy products, eggs, and goitrogenic vegetables, while they were allowed to eat lean red and white meat and fish food. After 21 days, all the patients showed a significant decrease in the levels of anti-TG Abs $(-40 \%, P<-0.013)$, anti-microsomal Abs $(-57 \%, P<0.000)$, and anti-TPO Abs $(-44 \%, P<0.029)$ (Figure 4).

Interestingly, in the experimental group, bioimpedance analysis showed a decrease not only in body weight and BMI, but also a reduction in fat mass (Figure 5).

\section{Discussion}

Recognition of a continuum of clinical phenotypes of autoimmune thyroid disorders and understanding their main pathophysiological mechanisms have led to a new classification of these conditions, identifying two types: 1) type 1 autoimmune thyroiditis (euthyroidism and presence of anti-thyroglobulin and anti-peroxidase Abs) and 2) type 2 autoimmune thyroiditis or chronic Hashimoto's disease (hypothyroidism with anti-thyroglobulin, antiperoxidase, and anti-microsomal Abs).

Now, it is important to mention that many previous studies in overweight adult patients were targeted only against the relationship between variations of thyroid hormone levels with respect to body weight, ignoring the presence and/or the levels of anti-thyroglobulin, anti-microsomal, and anti-peroxidase Abs, which eventually lead to Hashimoto's disease. In fact, in these studies, positive results in terms of weight reduction with respect to the diet regime implemented were reported, but any variations of the thyroid hormones and TSH were only mentioned. ${ }^{10-20}$ Ultimately, after 3 months,

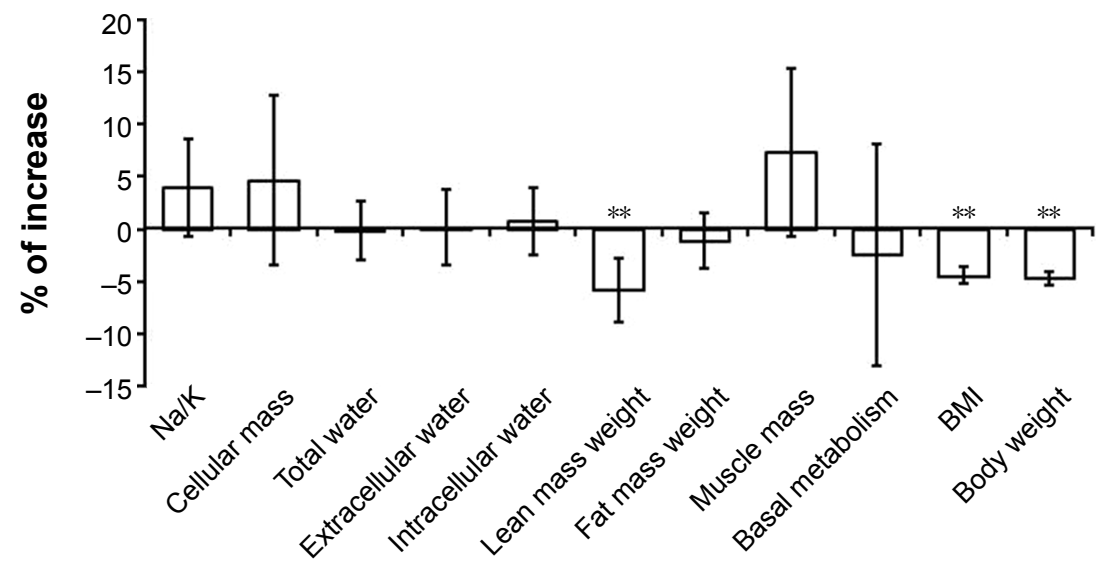

Figure 2 Patients following a normal diet, not deprived of carbohydrates, show significant variations of bioimpedance values (\%) of lean mass, BMI, and body weight. Note: **Significance of differences was set at $P<0.01$.

Abbreviation: BMI, body mass index. 


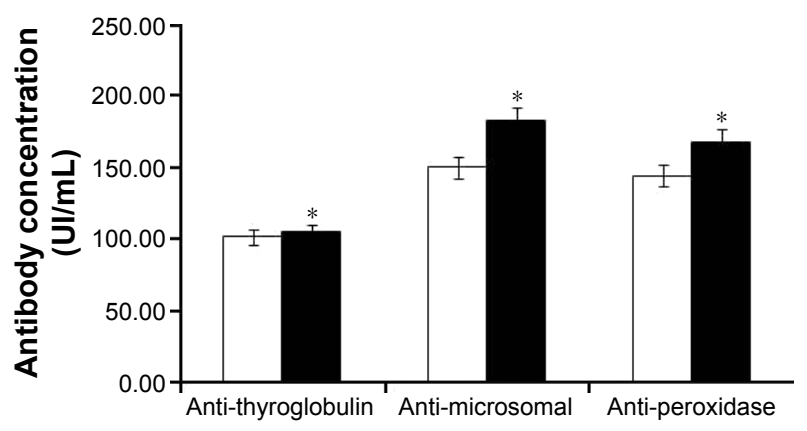

Figure 3 The value of autoantibodies in patients following a normal diet. Notes: The anti-thyroglobulin $\mathrm{Ab}$ and anti-thyroperoxidase or anti-microsomal $\mathrm{Ab}$ levels of TPO Ab showed a significant difference. Open bars indicate the patients at the beginning of experimental time, while the solid bars indicate values of patients after 21 days of the diet. *Significance of differences was set at $P<0.05$.

Abbreviations: $\mathrm{Ab}$, antibody; TPO, thyroperoxidase.

these patients regained weight and all their physical parameter values were again out of the normal range. In the current study, we implemented a diet plan based on a minor content of simple and complex carbohydrates, milk, dairy products, and legumes, but rich in animal proteins, that has allowed us to obtain at least two main objectives: a significant reduction in BMI and body weight and a highly significant reduction in autoantibody titers. By contrast, there were no significant reductions in the level of autoantibodies in the patients in the control group after following an unrestricted dietary regime. Fundamentally, the aim of this type of diet is to reduce the overall inflammatory state and consequently inflammation of the thyroid gland. ${ }^{21-29}$ It is therefore evident that an improvement in the general conditions of the patients undergoing an ad hoc dietary regime leads to both a reduction of body

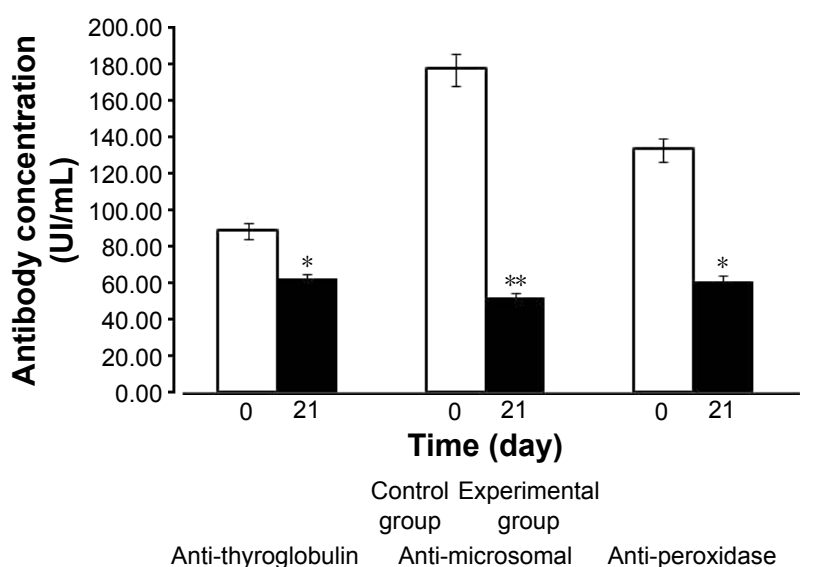

Figure 4 The value of autoantibodies in patients following a low-carbohydrate diet therapy.

Notes: The anti-thyroglobulin $\mathrm{Ab}$ and anti-thyroperoxidase or anti-microsomal $\mathrm{Ab}$ levels of TPO Ab showed a significant difference. Open bars indicate the patients at the beginning of experimental time, while the solid bars indicate values of patients after $2 \mathrm{I}$ days of the diet. $* * *$ Significance of difference was set at $P<0.05$ and $P<0.01$, respectively.

Abbreviations: Ab, antibody; TPO, thyroperoxidase. weight and levels of autoantibodies, and it would not be hazardous to hypothesize that these results are related to the role of the thyroid hormone receptor- $\alpha(\mathrm{TR} \alpha)$, found mainly in the liver and white adipose tissues. In fact, various studies have demonstrated the role of TR $\alpha$ in lipid metabolism and homeostasis. It has been shown that $\mathrm{TR} \alpha$ regulates the activity of LXR and SREBP-1c and the expression of ChREBP. ${ }^{30,31}$ It has also been suggested that TR $\alpha$ could be involved in the transcriptional regulation of lipogenesis in the liver. Activation of these genes determines an increase in the expression of anti-microsomal Abs, which occurs mainly in the REL of hepatocytes, and it has a fundamental role in inflammatory processes.

It is worth to note that $83 \%$ of patients with a high level of autoantibodies are breath test positive to lactase with a lactase deficit higher than $50 \%$ (personal communication). All these support our results, because the reduction of simple and complex carbohydrates in the diet, as we have done in our dietary plan, probably reduces the translocation of the ChREBP protein, a phenomenon necessary to induce transcription of genes involved in lipogenesis. In fact, a recent study reported that ChREBP is necessary to induce $L-P K$ and ACC glucose-dependent genes, suggesting the probable existence of other transcriptional regulators in the liver different from LXR. ${ }^{30-36}$

After all, glucose is not only a primary carbon source for anabolic and catabolic purposes, but also serves as a signal in the regulation of metabolic gene expression. In this sense, ChREBP is a transcriptional factor that responds to glucose signaling. ${ }^{37,38}$

ChREBP mRNA is most abundant in tissues in which lipogenesis is highly active..$^{39-42}$ In hepatocytes and adipocytes, ChREBP- $\alpha$ expression is induced by glucose. ${ }^{42-46}$

ChREBP regulates a high number of genes encoding glycolytic enzymes, most of the genes involved in NADPH generation, and genes that play roles in regulating metabolic and energy homeostasis. ${ }^{47,48}$ In summary, ChREBP/Mlx represents the principal transcription factor in the upregulation (in a glucose-dependent manner) of genes involved in glucose uptake, glycolysis, and lipogenesis in metabolic tissues such as the liver and brown and white adipose tissues.

Understanding the role of ChREBP in the various tissues will provide important insight into the pathogenesis of metabolic syndrome.

Chronic inflammation of the thyroid gland can also be due to the presence of environmental contaminants that are suspected to disturb activity against the endocrine system, known as endocrine disrupting chemicals. In fact, specific studies on thyroid hormone metabolism in female rats fed 


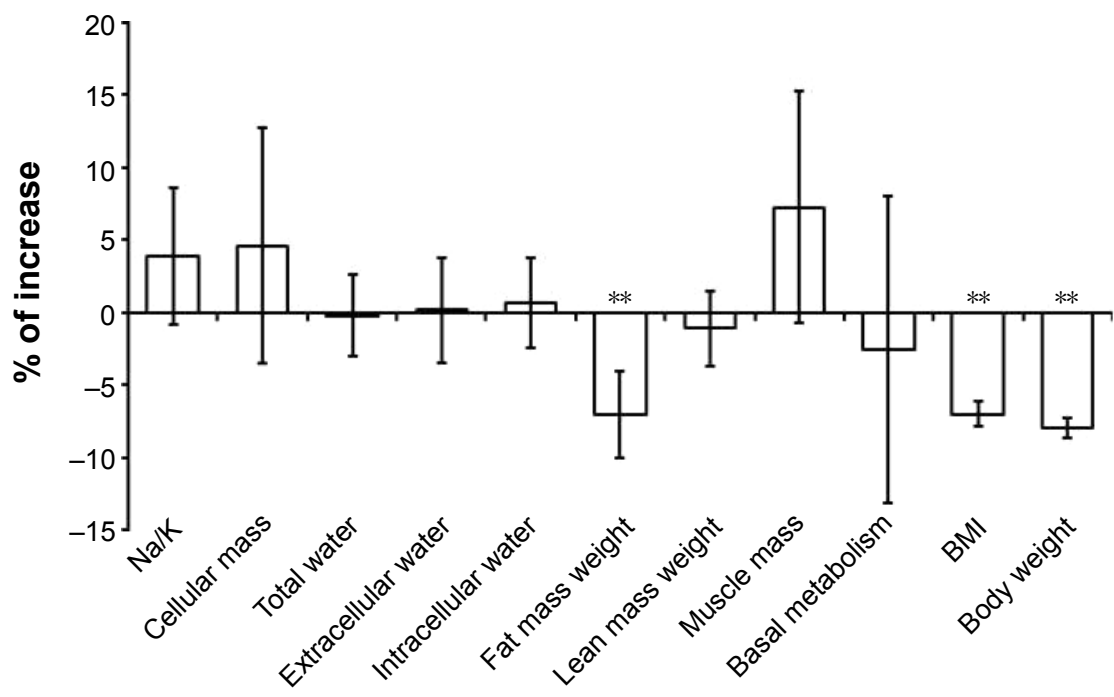

Figure 5 Patients following a diet devoid of carbohydrates show significant variations of bioimpedance values (\%) of lean mass, BMI, and body weight. Note: **Significance of differences was set at $P<0.05$.

Abbreviation: BMI, body mass index.

with diets containing TCDD, PCB126, and PCB156 at concentrations of PCB156 varying from 0.2 to $20 \mathrm{mg} / \mathrm{kg}$ have reported significant correlations between decreased concentrations of plasma thyroxine and Phase II microsomal UDP-glucuronyltransferase enzyme and also co-induction of Phase I and Phase II enzymes, such as CYP1a-P450; this clearly suggests the involvement of an Ah-mediated receptor in thyroid hormone metabolism disruption. ${ }^{49,50}$ Thus, a significant increase in anti-microsomal Abs in autoimmune thyroiditis could be interpreted as a compensation mechanism to avoid ulterior thyroid hormone degradation and consequently the rapid loss of thyroid function. The results of these studies and the results of our study will surely bring insight on the role of thyroid gland in the metabolism of lipids.

\section{Conclusion}

A dietary plan based on the reduction of carbohydrate content and free of goitrogenic foods leads not only to a decrease in body weight, but also determines a decrease in fat mass and a significant drop of sentinel autoantibodies in Hashimoto's thyroiditis. So, future studies could shed new light on the probable synergism between ChREBP-LXR, environmental contaminants, regulation of lipogenesis, and autoantibody production. Do not underestimate the value of the levels of anti-microsomal Abs, still not considered relevant in screening for autoimmune thyroiditis, the dietary regime described in this study could be implemented for the treatment of patients with autoimmune thyroid because of the possibility to reduce the inflammation state in general and of the thyroid gland in particular, and consequently of the levels of autoantibodies, information this surely important in the assessment of these patients and for the prediction of the course of the disease.

\section{Acknowledgments}

The abstract of this paper was presented at the 20th International Congress of Nutrition, Granada, Spain, September 15-20, 2013, as a poster presentation with interim findings. The poster's abstract was published in Ann Nutr Metab 2013;63(suppl 1):1-1960. This work is dedicated to Professor Anna Cardone, a dear friend who died prematurely. Publication realized with the contribution on the funds of $5 \times$ 1000 of IRPEF in favor of University of Foggia, in memory of Gianluca Montel.

\section{Disclosure}

The authors report no conflicts of interest in this work.

\section{References}

1. Tuzcu A, Bahceci M, Gokalp D, Tuzun Y, Gunes K. Subclinical hypothyroidism may be associated with elevated high-sensitive c-reactive protein (low grade inflammation) and fasting hyperinsulinemia. Endocr J. 2005;52(1):89-94.

2. Comitato R, Esposito T, Cerbo G, Angelini F, Varriale B, Cardone A. Impairment of spermatogenesis and enhancement of testicular germ cell apoptosis induced by exogenous all-trans-retinoic acid in adult lizard Podarcis sicula. J Exp Zool A Comp Exp Biol. 2006;305(3): 288-298.

3. Cardone A, Angelini F, Esposito T, Comitato R, Varriale B. The expression of androgen receptor messenger RNA is regulated by triiodothyronine in lizard testis. J Steroid Biochem Mol Biol. 2000;72(3-4): 133-141.

4. Fujita K, Okabe N, Yao T. Immunological studies on Crohn's disease. II. Lack of evidence for humoral and cellular dysfunctions. J Clin Lab Immunol. 1985;16(3):155-161. 
5. Esposito T, Lobaccaro J, Esposito M, Monda M, Varriale B. Carbohydrate deprivation and body weight in thyroid autoimmunity. Ann Nutr Metab. 2013;63:1200-1201.

6. Esposito T, Napoleone A, Allocca S, Varriale B, Monda M. Diet therapy of obesity: observations on the usefulness of weekly supervision in the improvement of weight loss. J Obes Weight Loss Ther. 2014;4:3.

7. Yu Y, Maguire TG, Alwine JC. ChREBP, a glucose-responsive transcriptional factor, enhances glucose metabolism to support biosynthesis in human cytomegalovirus-infected cells. Proc Natl Acad Sci U S A 2014;111(5):1951-1956.

8. Iizuka K. Recent progress on the role of ChREBP in glucose and lipid metabolism. Endocr J. 2013;60(5):543-555.

9. Benvenga S, Trimarchi F. Changed presentation of Hashimoto's thyroiditis in North-Eastern Sicily and Calabria (Southern Italy) based on a 31-year experience. Thyroid. 2008;18(4):429-441.

10. Tagliaferri M, Berselli ME, Calò G, et al. Subclinical hypothyroidism in obese patients: relation to resting energy expenditure, serum leptin, body composition, and lipid profile. Obes Res. 2001;9(3):196-201.

11. Monda M, Messina G, Mangoni C, De Luca B. Resting energy expenditure and fat-free mass do not decline during aging in severely obese woman. Clin Nutr. 2008;27(4):657-659.

12. Messina G, Vicidomini C, Viggiano A, et al. Enhanced parasympathetic activity of sportive women is paradoxically associated to enhanced resting energy expenditure. Auton Neurosci. 2012;169(2):102-106.

13. Messina G, Monda V, Moscatelli F, et al. Role of orexin system in obesity. Biol Med (Aligarh). 2015;7:4

14. Monda M, Viggiano A, Viggiano A, et al. Olanzapine blocks the sympathetic and hyperthermic reactions due to cerebral injection of orexin $\mathrm{A}$ Peptides. 2008;29(1):120-126.

15. Monda M, Viggiano A, Viggiano A, et al. Quetiapine lowers sympathetic and hyperthermic reactions due to cerebral injection of orexin A. Neuropeptides. 2006;40(5):357-363.

16. Monda M, Messina G, Vicidomini C, Viggiano A, Mangoni C, De Luca B. Activity of autonomic nervous system is related to body weight in pre-menopausal, but not in post-menopausal women. Nutr Neurosci. 2006;9(3-4):141-145.

17. Messina G, De Luca V, Viggiano A, et al. Autonomic nervous system in the control of energy balance and body weight: personal contributions Neurol Res Int. 2013;2013:639280.

18. Iacobellis G, Ribaudo MC, Zappaterreno A, Iannucci CV, Leonetti F. Relationship of thyroid function with body mass index, leptin, insulin sensitivity and adiponectin in euthyroid obese women. Clin Endocrinol (Oxf). 2005;62(4):487-491.

19. Zimmermann-Belsing T, Brabant G, Holst JJ, Feldt-Rasmussen U. Circulation leptin and thyroid dysfunction. Eur J Endocrinol. 2003; 149(4):257-271.

20. Spencer CA, Hollowell JG, Kazarosyan M, Braverman LE. National Health and Nutrition Examination Survey III thyroid-stimulating hormone (TSH)-thyroperoxidase antibody relationships demonstrate that TSH upper reference limits may be skewed by occult thyroid dysfunction. J Clin Endocrinol Metab. 2007;92(11):4236-4240

21. Samuels MH, Kolobova I, Smeraglio A, Peters D, Purnell JQ, Schuff KG Effects of levothyroxine replacement or suppressive therapy on energy expenditure and body composition. Thyroid. 2016;26(3):347-355.

22. D’Herbomez M, Wémeau JL. Revues générales Exploration de l'autoimmunité thyroïdienne: apport du laboratoire. Ann Biol Clin. 2001;59(6): 717-723.

23. Iizuka K, Horikawa Y. ChREBP: a glucose-activated transcription factor involved in the development of metabolic syndrome. Endocr J. 2008;55(4):617-624.

24. Denechaud PD, Dentin R, Girard J, Postic C. Role of ChREBP in hepatic steatosis and insulin resistance. FEBS Lett. 2008;582(1):68-73.

25. Messina G, Palmieri F, Monda V, et al. Exercise causes muscle GLUT4 translocation in an insulin-independent manner. Biol Med (Aligarh). 2015;1:2.

26. Messina G, Zannella C, Monda V, et al. The beneficial effects of coffee in human nutrition. Biol Med (Aligarh). 2015;7:4.
27. Di Bernardo G, Messina G, Capasso S, et al. Sera of overweight people promote in vitro adipocyte differentiation of bone marrow stromal cells. Stem Cell Res Ther. 2014;5(1):4.

28. Monda M, Messina G, Scognamiglio I, et al. Short-term diet and moderate exercise in young overweight men modulate cardiocyte and hepatocarcinoma survival by oxidative stress. Oxid Med Cell Longev. 2014; 2014:131024.

29. Duntas L, Hauner H, Rosenthal J, Pfeiffer EF. Thyrotropin releasing hormone $(\mathrm{TRH})$ immunoreactivity and thyroid function in obesity. Int J Obes. 1991;15(1):83-87.

30. Viennois E, Esposito T, Dufour J, et al. LXR $\alpha$ regulates the androgen response in prostate epithelium. Endocrinology. 2012;153(7): 3211-3223.

31. Reinehr T, Isa A, de Sousa G, Dieffenbach R, Andler W. Thyroid hormones and their relation to weight status. Horm Res. 2008;70(1): $51-57$.

32. Triggiani AI, Valenzano A, Ciliberti MA, et al. Heart rate variability is reduced in underweight and overweight healthy adult women. Clin Physiol Funct Imaging. 2015. doi:10.1111/cpf.12281.

33. Valenzano A, Moscatelli F, Triggiani AI, et al. Heart-rate changes after an ultraendurance swim from Italy to Albania: a case report. Int J Sports Physiol Perform. 2016;11(3):407-409.

34. Rinaldi B, Guida F, Furiano A, et al. Effect of prolonged moderate exercise on the changes of nonneuronal cells in early myocardial infarction. Neural Plast. 2015;2015:265967.

35. Messina G, Dalia C, Tafuri D, et al. Orexin-A controls sympathetic activity and eating behavior. Front Psychol. 2014;5:997.

36. Postic C, Dentin R, Denechaud PD, Girard J. ChREBP, a transcriptional regulator of glucose and lipid metabolism. Annu Rev Nutr. 2007;27: 179-192.

37. Uyeda K, Repa JJ. Carbohydrate response element binding protein, ChREBP, a transcription factor coupling hepatic glucose utilization and lipid synthesis. Cell Metab. 2006;4(2):107-110.

38. Viggiano A, Chieffi S, Tafuri D, Messina G, Monda M, De Luca B. Laterality of a second player position affects lateral deviation of basketball shooting. J Sports Sci. 2014;32(1):46-52.

39. Iizuka K, Bruick RK, Liang G, Horton JD, Uyeda K. Deficiency of carbohydrate response element-binding protein (ChREBP) reduces lipogenesis as well as glycolysis. Proc Natl Acad Sci U S A. 2004;101(19): 7281-7286.

40. Viggiano AN, Vicidomini C, Monda M, et al. Fast and low-cost analysis of heart rate variability reveals vegetative alterations in noncomplicated diabetic patients. J Diabetes Complications. 2009;23(2):119-123.

41. Monda M, Viggiano A, Viggiano A, et al. Sympathetic and hyperthermic reactions by orexin A: role of cerebral catecholaminergic neurons. Regul Pept. 2007;139(1-3):39-44.

42. Dentin R, Pégorier JP, Benhamed F, et al. Hepatic glucokinase is required for the synergistic action of ChREBP and SREBP-1c on glycolytic and lipogenic gene expression. J Biol Chem. 2004;279(19): 20314-20326.

43. Esposito T, Tammaro P, Paolisso G, Varriale B. Hormonal regulation and characterization of MHG30 gene, a desaturase-like gene of hamster Harderian gland. J Steroid Biochem Mol Biol. 2015;154:267-273.

44. Esposito T, Varriale B, D'Angelo R, Amato A, Sidoti A. Regulation of flavin-containing mono-oxygenase (Fmo3) gene expression by steroids in mice and humans. Horm Mol Biol Clin Investig. 2014;20(3): 99-109.

45. Cardone A, Angelini F, Esposito T, Comitato R, Varriale B. The expression of androgen receptor messenger RNA is regulated by tri-iodothyronine in lizard testis. J Steroid Biochem Mol Biol. 2000;72(3-4):133-141.

46. He Z, Jiang T, Wang Z, Levi M, Li J. Modulation of carbohydrate response element-binding protein gene expression in 3T3-L1 adipocytes and rat adipose tissue. Am J Physiol Endocrinol Metab. 2004; 287(3):E424-E430.

47. Ma L, Robinson LN, Towle HC. ChREBP*Mlx is the principal mediator of glucose-induced gene expression in the liver. J Biol Chem. 2006;281(39):28721-28730. 
48. Van Birgelen AP, Smit EA, Kampen IM, et al. Subchronic effects of 2,3,7,8-TCDD or PCBs on thyroid hormone metabolism: use in risk assessment. Eur J Pharmacol. 1995;293(1):77-85.

49. Esposito M, Serpe FP, Diletti G, et al. Serum levels of polychlorinated dibenzo-p-dioxins, polychlorinated dibenzofurans and polychlorinated biphenyls in a population living in the Naples area, southern Italy. Chemosphere. 2014;94:62-69.
50. Esposito T, Viggiano A, Viggiano A, et al. ICV injection of orexin A induces synthesis of total RNA and mRNA encoding preorexin in various cerebral regions of the rat. $J$ Therm Biol. 2006;31:527-532.

\section{Publish your work in this journal}

Drug Design, Development and Therapy is an international, peerreviewed open-access journal that spans the spectrum of drug design and development through to clinical applications. Clinical outcomes, patient safety, and programs for the development and effective, safe, and sustained use of medicines are the features of the journal, which has also been accepted for indexing on PubMed Central. The manuscript management system is completely online and includes a very quick and fair peer-review system, which is all easy to use. Visit http://www.dovepress.com/testimonials.php to read real quotes from published authors.

Submit your manuscript here: http://www.dovepress.com/drug-design-development-and-therapy-journal 